\title{
Drawing Interfaces: When Interaction Becomes Situated and Variable
}

\begin{abstract}
The widespread and pervasive presence of devices equipped with small-size, small-cost sensors and increasing computational capacity affected our interactions, making them growingly ubiquitous and dynamic; an interaction that shifted from being indirect to becoming more physical and direct, as using gestural or vocal commands to control smart systems. In this context, we developed interfaces that draw themselves accordingly to the user need. Drawing User Interfaces receive information from the physical world (users and environment) via sensors, and react modifying their aesthetics and function.
\end{abstract}

\section{Keywords}

User Experience, User Interface, Physical Interaction, Embedded Technology, Smart Objects

\section{Interfaces, Embodiment and Materiality}

We are in a historical moment of change, where our way of interacting with objects is culturally evolving, due to the unceasing advances of technology. The progressive technological pervasiveness made a growing number of devices more intelligent, interactive and capable of dialoguing among themselves, with the surrounding context and with us. The result is innovative and advanced interactions that produce models and patterns of use that reinterpret the relationship between humans and technology [1-3]. In a context where people and computers are no more considered as separate but as a whole [4], our interest concerns integrated ecosystems where the physical and digital worlds dialogue because of embedded sensors, microcontrollers, and actuators that make machines "sensitive" to external stimuli $[5,6]$. Benefitting of a progressive increase in computational capacity (ubiquitous computing), such ecosystems can adapt automatically to circumstances and decrease users' cognitive load [7], namely the information they must process to perform a task. Overcoming Graphical User Interfaces (GUI), it expanded the range of possible interactions: from typing and visualizing (as PCs) to manipulation (as Beosound Edge that activates a touch-sensitive control interface when the user approaches), dialogue (as Amazon Alexa and Google Home that can be controlled starting a conversation through voice interaction), and gestures (as Xbox Kinect that responds to motion sensing inputs, making the user's body become the controller). This brought to a dual technological advance: the research on Natural User Interfaces (NUIs) [8, 9] and on Tangible User Interfaces (TUIs) [10]. In the former case, the logic revolves around the absence of a visible interface 
[11], namely user interfaces that are invisible to its users, or become so through subsequent interactions. The latter case regards embedding the interface into the object using manipulation logics typical of the analogical dimension [5, 12]. Although we recognize that NUls' immediate and non-mediated interactions are interesting and promising, TUls pop up as even more fascinating since they imply analog-like manipulations on objects that hide a digital dimension and echo the concept of memory of use [13]. However, dealing with physical and computational elements that empower materials, making them reactive and able to inform [14], opens up inevitable reasoning on materiality [15], embodiment $[16,17]$ and affordances $[1,18]$. TUls make the object on which are mounted both a representation of information and a controller [10]. Achieving such a total unity between interface and interaction has implications as the graspability of the interactions and actions supported [19]. Moreover, as stated by Ishii himself when criticizing tangible bits in favour of his more contemporary concept of radical atoms [14], TUls have limitations in representing change.

Then, recalling that objects are nowadays more than ever shapers of behavior [20], we cannot neglect that blending the physical and digital dimensions reconfigures the users' behaviour towards the product, impacting on the kind of learning such objects demand to be properly used [21]. The interfaces we propose advance from this very argument.

\subsection{Interfaces that Draw Themselves}

We consider that especially the material perspective brings interesting opportunities for the interaction design and $\mathrm{HCl}$ fields. Looking for potentially meaningful aesthetic interactions, our specific interest goes on the role that materials, environments and the user's physical body as a combination assume in interactions involving embodied technologies [16, 22]. From this notion, we developed what we define Drawing User Interfaces (DUIs henceforth), namely interfaces that draw themselves. Such interfaces receive information from the physical world (users, the environment, and potentially other objects) through sensors, and act accordingly, modifying their aesthetics and function. DUls concern interactions with objects where the digital information is translated into a manifestation that allows direct manipulation.
However, it differs from Ishii's radical atoms [14] where digital information assumes a physical form. Indeed, whereas the physical interaction is maintained, it goes through a visual interface that rises from the surface. DUls appear on objects rather than being the object itself or part of it, as in the case of TUls [10]. As GUls, they display the information on screens, but not the traditional ones. The interface is integrated into the same material that composes the devices, and the feedback is given by the very skin of the object that changes depending on the information they receive or to explain the result of an operation. The interface is embedded in the material, and what changes is either its chemical or physical properties. Designing interfaces that draw themselves implies to alter and modify users' perception of objects. In so doing, these interfaces advance a reasoning that grounds on hacking objects' meanings [23].

\section{Process and Methods}

The research is conducted by Thingk, a spin-off of the Politecnico di Milano that experiments with interactive technologies, designing products that disguise themselves. It digs into apparently simple and minimal artifacts (form) that are technologically enriched (function), following the slogan: "objects of daily use with superpowers" (thingk.design). Thingk takes advantage of the principles of digital transformation [24] while pursuing technological hybridization, blending analogue properties and digital immateriality. This approach results into objects with invisible technology and essential aesthetics, that are augmented with unexpected properties. By redesigning their appearance and functions, hence affordances and interactions, the meaning of the artefacts gets affected. Our study capitalizes on how different skins activate different functions, changing how they are interpreted and approached by user $[25,26]$.

Experimenting on how to use and innovate with DUls, we apply an iterative design process (fig. 1) where multiple approaches and methodologies are combined to answer different needs [21]. The overall process consists of two main macro-phases. The first follows a design-driven innovation concept [27] that is applied to the research and design phases, when prosumers are involved in co-design sessions with early prototypes 


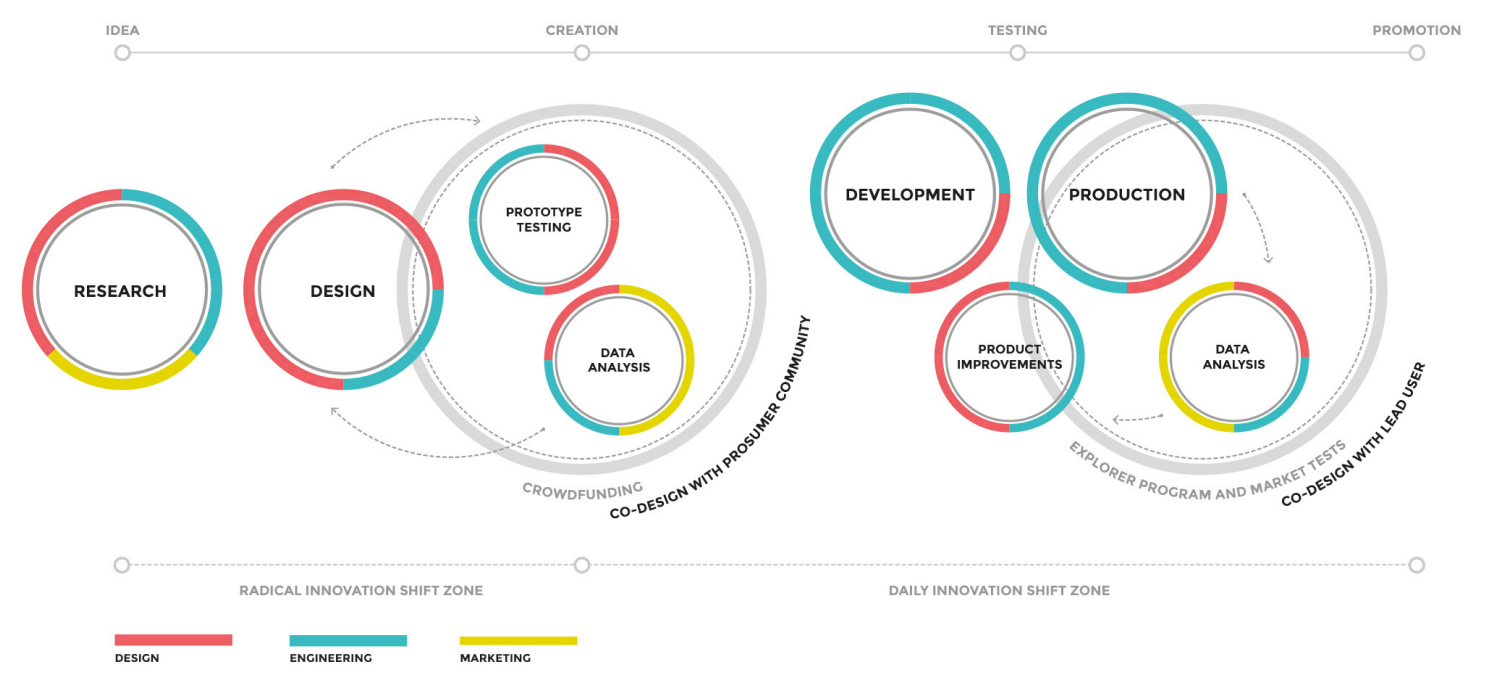

Fig. 1. Thingk's iterative design process, highlighting the steps and perspectives involved

to provide insights. The latter revolves around user testing and data analysis and regards development and production.

The Research phase focused on deepening our knowledge about contexts where several elements are intertwined, interacting among them and with the surrounding environment. We analysed lifestyle and technological trends (state of the art) to point out potentialities and constraints of the context of reference, especially breakthroughs in the contiguous areas of interaction design, electronics, and material research. Acknowledging the existence of interfaces that emerge from the surface as Mui's Calm Design, and our own Slab!, we are also aware of their limits, as their being static and localized. This suggested the concept of a multilayered interface in which layers are activated according to the function required. We tapped into extending from multiple functions to multiple language and meanings that coexist under the skin of the same object.

We are currently running the Design phase, with prototypes of use expected by the end of this year. Informed by the research conducted, we decided to completely hide the interface until the object detects motion, an interaction, or a connection with other objects. At this point, the interface appears. This phase involves lead users to verify their response towards the product/interfaces designed, gathering data from survey and qualitative enquiry (from rapid ethnography and participant observation to interviews), but also focus groups and workshops where sketches, renders and use cases are leveraged to stimulate discussion and validate our design choices. The Development and Production phases are expected to respectively start in one and two years. From an engineering and design perspective, the challenge is developing objects that reconfigure their own function according to the surface that they show, in a logic of quick adaptability to the context of use. We are currently conducting studies on possible technologies (electrochromic displays) and application (from smart product to smart buildings), as part of DecoChrom, a Horizon 2020 Project that will end in 2021. During the project we will verify DUls flexibility through three different applications on everyday objects. Working prototypes will be tested with the DecoChrom partners and a with community of prosumers, conducting user analysis to identify possible improvements.

\section{Results}

In conceptualizing the meaning of design, Krippendorff [25] described it as the attribution of meaning to things. Artifacts should, in fact, communicate their function through their aesthetic, which should introduce users to their correct functioning [28]. As theorized by Tidwell [29] the interface has an operational function that triggers the dialogue with the user. Therefore, redesigning the meaning of objects is a practice of a certain importance, which becomes paramount if we consider that Thingk reframes the 
form-function relationship of products, pursuing an innovation inspired by design, while recognizing users' needs [26], and the current growing technological opportunities [27]. When objects change their meaning, their language becomes anything but obvious [30]. Examples are those interactive artefacts that rely on integrated technological component (IoT) to become extended systems that dialogue with applications and other objects. Starting from such assumptions, we conceptualized a model of variable and situated interfaces that reacts not just to the interactions triggered by users, but also to the surrounding environment (context of use). Recognising the limitations and constraints of both TUls [14] and NUIs [8], and being aware of the invisibility dilemma [31] that comes along, we conceived a typology of dynamic interface that expresses itself and communicates to the user what its form hides (affordance). In an attempt to integrate smart functionality into everyday objects without falling into the aforementioned dilemma of choosing between minimizing disturbance from the main task and adding value including explicit interaction, there is a tendency to hide object's intelligence.

However, the consequence of keeping/making invisible to the user such an increase of functionality results into a dichotomy between aesthetics and functions. Making functionalities recognisable allows users to identify such augmented artifacts as smart and hence use them appropriately. In parallel, the control dilemma [32] exposes a crucial point when dealing with smart object automation. The attention lies in designing objects that act and react autonomously to certain inputs, hiding the ongoing complexity to the user without lessening his/her perception of applying control. On this regard, significant is the study on the use of the remote control Nest Learning Thermostat, where the users' interviewed stated they were not able to fully understand the object learning process of their habits, hence they were not able to utterly rely on how the smart home automation device was self-setting certain functionality. The control dilemma requires to pay attention to the user, who wants to know what is going on; this insight is on the ground of DUls concept.

\subsection{Ecosystemic (Situated) User Experiences}

Focusing on functionality, we often face the problem of how much information an interface should return in order to perform each task. The advantage lies on using a different set of variable commands that allows to isolate and "situate", or contextualize, a device's main functions according to its actual use case. Employing DUls allows indeed to define situated user experiences that are variable in time and space since they are closely related to the context in which they are immersed. Moreover, these interfaces can be modified and updated over time, potentially changing the product functionality: the object doesn't change, it updates its functions. In this perspective, an object or device can change the interface depending on, for example, its own positioning, and inclination, or according to the location of the user.

So far, we identified three possible use cases:

- Movement: Response to being relocated within an environment

- Reaction: Response to environmental changes (presence of people, physical variations, and other modification in or of the context)

- Dialogue: Functional variation based on the relationship with other connected objects present in the surrounding space

Below we present existing case studies as pretexts to explain possible use cases of DUls. Describing their functioning we explain how DUls could be applied to perform similar functions, making the interaction more direct, complete and immediate. In the first use case, Movement, the interface responds to the change of position and orientation, revealing itself and displaying different functions. Microsoft' Surface Dial is a controller that interacts with different programs and digital elements, serving specific functions according to how it is activated and manipulated. However, the function of the interface is delegated to an external screen, where the information is displayed, while the product itself does not provide any visual feedback on its surface. In this case study, the potential use of DUIs lies in overlapping object and interface, so that functions are communicated directly on the product. In the second use case, Reaction, the interface reacts to proximity or interaction with external elements, which can be as much the user as the environment with its variables. Lapka's Environment consists of a series of four environmental sensors embedded into as much small 


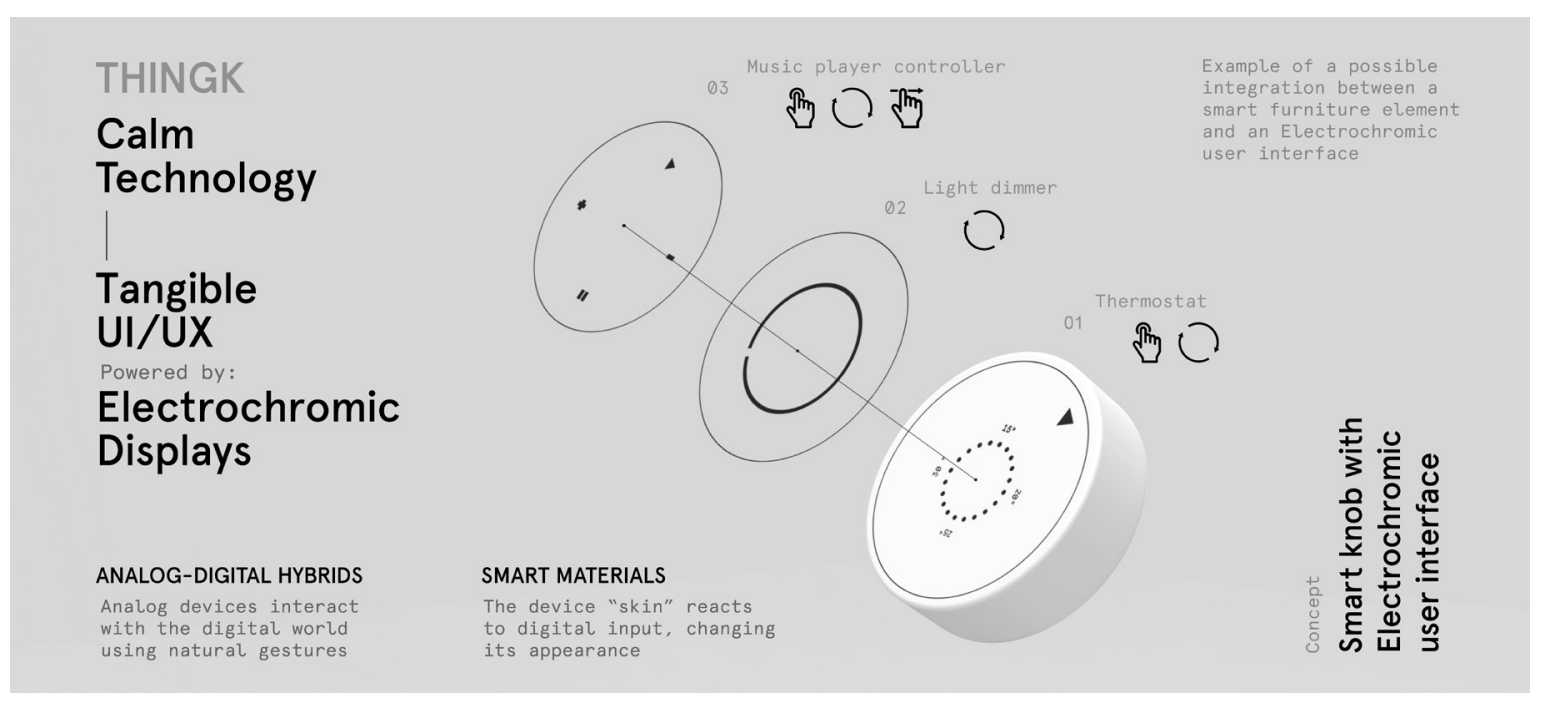

Fig. 2. Drawing User Interfaces: concept of a possible integration of functions

devices, translating complex data into user-friendly visualizations displayed on the smartphone. Once again, the function of showing data is entrusted to an external element: the smartphone display. Mounting DUls could allow to provide immediate feedback on the product itself. In the last use case, Dialogue, the interface relates to the surrounding smart objects. Ikea's Trådfri remote control connects to different lamps working by proximity, managing on and off, dim, and color temperature. The remote control recognizes and regulate bulbs within a range of $10 \mathrm{~m}$, but does not provide information or feedback. In this case, applying DUls would allow to show the object to which the remote control is connected, clarifying the functions available. In addition, in the hypothesis of multiple smart objects in the surrounding environment that could be managed by a single controller, DUls could change the controller interface depending on the object ruled and the function that can be performed. Changing its communicative skin, the object performs tailored functions depending on the interpretation of the surrounding variables. The research phase brought to think to functional layers that overlap, populating a single object with multiple capabilities. The main result of our study is designing the transition among various commands and as many functions that coexist in the same object. The output of our investigation is a surface capable of smoothly changing and reacting during the user experience.

To provide a sharp idea of how they work, fig. 2 presents a use case of DUls applied on a smart object acting as a controller. The object can be exemplified as a cylinder embedding smart sensors that can perform the regulation of values as temperature, volume, and so on, and that is provided with a silent interface. Such object contains multiple layers, each with its own function that gets activated according to the previously described use cases. The object becomes a repository of information that is going be communicated once used. For example, one layer could show how to regulate the temperature through rotation and selection; another one could display a linear scale to operate on the intensity of light, using rotation to dimmer; then, the third layer could allow to play/manage music or movies through interactions as selection, rotation and swipe. This design scenario is an example of possible use cases, as employing DUls to increase the variables at play, introducing an auto-nomatic dimension [33] that enhances the qualities of the elements involved. Attributing a performing ability to the interface, its visual elements gain the ability to instantly act and react to manipulation [34]. These feature and dynamicity require to design considering the relation between the object and the environment both in the form of positional/static identities and as variable elements that are fluid and responsive in their composition.

\section{Discussion. A Discourse on Possibilities and Constraints}

The use cases described can be developed harnessing various technology. The ongoing tendency to dematerialise objects' physical interfaces frequently 
led to delegate the operational functions of an object to digital screens. Indeed, most of the contemporary smart products uses embedded displays or transfer data to other devices with screens (mobile and tablet). Such displays, acting as communication gateway, are in charge of returning information. That said, the current technological state of the art witnesses the appearance of smart materials able to reconfigure their shape, becoming potential drivers of information. However, we are still far from the concrete possibility of a material so ductile to display a continuous and updated flow of digital data, through a modification of its intrinsic physical properties. At this state of technology, DUls are affected by this restriction, and they present a further limitation: since they allow an object to embed multiple functionalities, they do not make any function manifest by default. The absence of the interface, when none of the layers is active, as well as the presence of a variable interface concealed until the moment of use, affects the way in which the object communicates and is interpreted by the user.

In addition, their being entirely based on visual elements makes them unsuitable for being used by people with visual disabilities. By contrast, the information behind these visual components could be translated or implemented by combining them with additional sensory stimuli. For example, dealing with TUls, Ishii recommends the use of more "malleable" forms of feedback, such as audio and video, that complement, support and complete interfaces. Our reasoning on DUls possible implementations goes in a direction similar to what Ishii [10] identified as Double Interaction Loop, namely a cycle that starts with a first and immediate level of interaction and haptic feedback provided in consequence of touching, grasping and manipulating a physical object, followed by intangible feedback (as audio) and possible physical modification of the object, reflecting a change of the digital data. A possible field of application of DUls is between analog (aesthetics and materials) and digital (functions and transitions). Several technologies can be used to build such interfaces. Particularly, we investigate those materials that change the state of their chemical (electrochromic) or physical (e-ink) properties. Both technologies can be integrated into surfaces made of natural materials - as wood, marble, metal-without contaminating their appearance and tactile properties. On the one hand, aiming at the e-ink, as in the case of the Yotaphone, ensures excellent graphic resolution but has a color range limited to black and white. On the other hand, electrochromic screens have the advantage of being the result of a monochrome screen printing technique on transparent surfaces, allowing them to be positioned above the material of the object itself. However, since electrochromic is the technology subject of the research of the European project DecoChrom, it is currently the basis on which we are conducting the experimentation. An advantage of choosing this not-new technology is that the electrochromic absorbs energy only when transitioning from a state of transparency to that of opacity; once a state is reached, there is no consumption related to its maintenance.

Designing interfaces we follow a model of experimentation closely connected to the field of loT where the relationship between objects, technology and context is at the heart of the project. We privilege a design-driven innovation method that takes into account the needs of the user. Hence, the innovation is oriented to the relationship between function and aesthetics, and it relates to the context of use. One of the results achieved is to preserve the aesthetics of products that historically did not mount interfaces, inserting an interactive layer capable of manifesting itself and disappearing when needed. The implications of integrating physical elements with computational processes expand the design possibilities in terms of choosing materials that become reactive, dynamic and able to shape, transform and inform [13]. We consider this reasoning significant to various disciplines, from design to $\mathrm{HCl}$, as it explores how interfaces can exploit smart technologies to modify themselves while mixing digital and physical.

As said, DUls are currently in the prototyping phase, as user research will take place in 2020. The main issue that will be the subject of our upcoming enquire concerns how users will react in front of interfaces that design themselves when necessary, namely changing according to their being placed in space, the dialogue with other smart objects of the digital ecosystem, or the interaction with the user. In doing so, it is central to 
understand the role of aesthetics in dynamic digitalphysical ecosystems. Addressing the design of interfaces for distributed, hyperconnected, and complex smart ecosystem requires to tap into the implications of having multiple meanings coexisting into the same object; meanings that are not persistently displayed, but emerge from the surface when needed. In consequence, it is crucial to enquire the way in which the users will interpret and interact with objects that contain interfaces that are simultaneously:

- Hidden, since they are not always displayed

- Variable, since they reconfigure themselves according to the environment

- Updatable, since they are designed to last more time than the lifespan of the object itself.

To conclude, DUls take advantage of how microprocessors and sensors can provide an ecosystem of smart objects with awareness of their surrounding, exploiting their ability to dialogue with users, other objects and the environment. These interfaces can disappear, hence they can be used on objects and products that maintain their overall aesthetics while being augmented. The interface is no more influencing or contaminating the look-and-feel of a product. That said, future developments concern the enquiry on users to understand how such interfaces have implications in terms of experience; in parallel, possible advances of electrochromic technology, as well as the application of other technologies, could positively impact on the project.

\section{References}

1. Latour, B.: On interobjectivity. Mind, culture, and activity. 3(4), 228-245 (1996).

2. Jenkins, T., Bogost, I.: Designing for the internet of things: prototyping material interactions. In: CHI'14 Extended Abstracts on Human Factors in Computing Systems. pp. 731-740. ACM (2014).

3. Evans, D.: The internet of everything: How more relevant and valuable connections will change the world. Cisco Internet Business Solutions Group (IBSG). 2012, 1-9 (2012).

4. Wiberg, M., Robles, E.: Computational compositions: Aesthetics, materials, and interaction design. International Journal of Design. 4(2), 65-76 (2010).

5. Shaer, O., Hornecker, E., others: Tangible user interfaces: past, present, and future directions. Foundations and Trends $®$ in Human-Computer Interaction. 3(1), 4-137 (2010).

6. Vermesan, O., Friess, P.: Internet of things: converging technologies for smart environments and integrated ecosystems. River publishers, Delft, Netherlands (2013).

7. Sweller, J.: Cognitive load theory. In: Psychology of learning and motivation. pp. 37-76. Elsevier, New York, NY (2011).

8. Norman, D.A.: Natural User Interfaces are not Natural. Interactions. 17(3), 6-10 (2010).

9. Ballmer, S.: CES 2010: A Transforming Trend — The Natural User Interface, www.huffingtonpost.com/steve-ballmer/ces2010-a-transforming-t_b_416598.html.

10. Ishii, H.: Tangible bits: beyond pixels. In: Proceedings of the 2nd international conference on Tangible and embedded interaction. pp. $\times v-\times \times v$. ACM (2008).

11. Krishna, G.: The best interface is no interface: the simple path to brilliant technology. New Riders, San Francisco, CA (2015).

12. Wigdor, D., Wixon, D.: Brave NUI world: designing natural user interfaces for touch and gesture. Elsevier, New York, NY (2011).

13. Wilson, F.R.: The hand: How its use shapes the brain, language, and human culture. Vintage, New York, NY (1999).

14. Ishii, H., Lakatos, D., Bonanni, L., Labrune, J.-B.: Radical atoms: beyond tangible bits, toward transformable materials. Interactions. 19(1), 38-51 (2012).

15. Pizzocaro, S.: Matter still matters: design education for a material culture in the immaterial age. Temes de Disseny: nueva etapa. 92-103 (2018).

16. Dourish, P.: Where the action is: the foundations of embodied interaction. MIT Press, Cambridge, MA (2004).

17. Fernaeus, Y., Tholander, J., Jonsson, M.: Towards a new set of ideals: consequences of the practice turn in tangible interaction. In: TEl'08 Proceedings. pp. 223-230. ACM (2008).

18. Gibson, J.J.: The ecological approach to visual perception. Houghton Mifflin, Boston, MA (1986).

19. Jacob, R.J., Girouard, A., Hirshfield, L.M., Horn, M.S., Shaer, O., Solovey, E.T., Zigelbaum, J.: Reality-based interaction: a framework for post-WIMP interfaces. In: Proceedings of the SIGCHI conference on Human factors in computing systems. pp. 201-210. ACM (2008).

20. Kolko, J.: Thoughts on interaction design. Morgan Kaufmann, Burlington, MA (2010).

21. Saffer, D.: Designing for interaction: creating innovative applications and devices. Peachpit press, Berkeley, CA (2010). 
22. Hornecker, E., Buur, J.: Getting a grip on tangible interaction: a framework on physical space and social interaction. In: Proceedings of the SIGCHI conference on Human Factors in computing systems. pp. 437-446. ACM (2006).

23. Tolino, U., Mariani, I.: Hacking Meanings. Innovation as Everyday Invention. DIID Disegno Industriale. Design as inventor. 65(18), 92-99 (2019)

24. Kuniavsky, M.: Smart Things, Ubiquitous Computing User Experience Design. Elsevier, Burlington, MA (2010)

25. Krippendorff, K.: On the Essential Contexts of Artifacts or on the Proposition That "Design Is Making Sense (of Things)." Design Issues. 5(2), 9-39 (1989).

26. Heskett, J.: Toothpicks and logos: Design in everyday life. Oxford University Press, Oxford, UK (2002).

27. Utterback, J., Vedin, B., Alvarez, E., Ekman, S., Walsh Sanderson, S., Tether, B., Verganti, R.: Design-inspired innovation and the design discourse. Design-inspired innovation. 154-186 (2006).

28. Norman, D.A.: Affordance, Conventions, and Design. Interactions. 6(3), 38-43 (1999).

29. Tidwell, J.: Designing interfaces: Patterns for effective interaction design. O'Reilly Media, Sebastopol, CA (2010).

30. Tolino, U., Mariani, I.: Do You Think What I Think? Strategic Ways to Design Product-Human Conversation. Strategic Design Research Journal. 11(3), 254-262 (2018).

31. Kranz, M., Holleis, P., Schmidt, A.: Embedded interaction: Interacting with the internet of things. IEEE internet computing. 46-53 (2009).

32. Yang, A., Rebaudengo, S.: Yang, R., Rabaudengo S.: When Objects Talk Back, https://medium.com/@frogdesign/whenobjects-talk-back-8eff3b252482.

33. Botta, M., Smith, G.C., Anceschi, G.: Design dell'informazione: tassonomie per la progettazione di sistemi grafici auto-nomatici. Valentina Trentini, Trento, Italy (2006).

34. Tolino, U.: Figure dinamiche e di interazione. In: Bucchetti, V. (ed.) Progetto e culture visive. Elementi per il design della comunicazione. FrancoAngeli, Milano, Italy (2018). 